\title{
An Update on the Progress in High-n Immersion Lithography
}

\author{
Harry Sewella, Paul Graeupner ${ }^{\mathrm{d}}$, Diane McCafferty ${ }^{\mathrm{a}}$, Louis Markoya a , Nandasiri \\ Samarakone $^{c}$, Paul van Wijnen ${ }^{b}$, Jan Mulkens ${ }^{b}$, and Jos Benschop ${ }^{b}$ \\ ${ }^{a}$ ASML, 77 Danbury Rd. Wilton, CT06897, USA; \\ ${ }^{b} A S M L$, De Run, Veldhoven, Netherlands; \\ ${ }^{c}$ ASML, Sunnyvale, California, USA.; \\ ${ }^{d}$ Zeiss-SMT, Oberkochen, Germany.
}

High-n immersion lithography has been under development for the past three years, the target being to extend Optical Lithography to the $32 \mathrm{~nm}$ node and beyond. Feasibility studies have been generating results on all aspects of the technology. This paper reports results obtained in key areas: High-n Immersion Fluid performance and lifetime; High-n Final Lens material development; Defect generation and control.

High-n Immersion Fluids have been developed by companies such as DuPont and JSR. The fluids typically have a refractive index of 1.65 and will support a system numerical aperture of $>1.55$. These new fluids have been engineered to a point where they are now more transparent than water at the exposing wavelength of $193 \mathrm{~nm}$, but challenges remain. Test results are reported from the UV The studies indicate that the UV irradiation of the fluid at high doses causes a build up of carbonaceous contamination on the final lens element surface. Cleaning procedures have been developed and calculations of the expected cleaning schedule made.

A key requirement for this technology is the availability of high refractive index final lens element material. Final lens element material must have a refractive index $>1.70$ to support optics design with $>1.55 \mathrm{NA}$; it must also have sufficient optical transparency. The availability of viable high-n immersion fluid means that the supply of optical grade high-n material for the final lens element fabrication is the potential technology show stopper. High-n materials $(>1.9)$ have been identified. Initial technical progress with materials such as LuAG and Spinel has been encouraging; the focus has been on obtaining starting materials that are pure enough to ensure a final optical material with a transparency that is high enough at $193 \mathrm{~nm}$ wavelength. There is, though, a risk that availability of optical grade material will miss the window for the technology.

Defect generation is another major concern with this Immersion Lithography. We have studied the effect on defect generation of using the high-n immersion fluid instead of water. There are defect generating mechanisms specific to the use of high-n immersion fluid. High-n immersion fluid is much more difficult to purify than water, and the purification process can generate particles which must be removed.

The new high-n immersion lithography can be assessed with regard to the Semiconductor Industry roadmap. The relatively slow development of high-n optical material implies that the potential for the technology now lies in the context of Double Pattern Processing for application to sub-32nm nodes.

Keywords: LuAG, High-n Immersion, 193nm Lithography, Immersion Defects.

\section{Introduction}

A three-year feasibility study looked at all aspects of building a High-n immersion lithography tool that would extend single exposure optical lithography beyond $38 \mathrm{~nm}$ and down to the $32 \mathrm{~nm}$ node. A tool which, in conjunction with double patterning, has the potential to reach the $16 \mathrm{~nm}$ node. 
Study subjects under review are in four major categories: the exposure system and fluid containment; fluids; the optics; and the wafer processing. This paper reports results from all four categories.

\section{High-n Immersion System Concepts}

Water-based immersion tools currently operate with the design goal of keeping the wafer dry of water, both before, and after, the wafer is passed under the stationary immersion system showerhead. This goal is achieved by the properties of the immersion water itself. Water has a high surface tension and a high contact angle to the resist or topcoat surface on the wafer. Water is therefore relatively easily constrained under the showerhead, even when the wafer is scanned at speeds of $600 \mathrm{~mm} / \mathrm{sec}$.

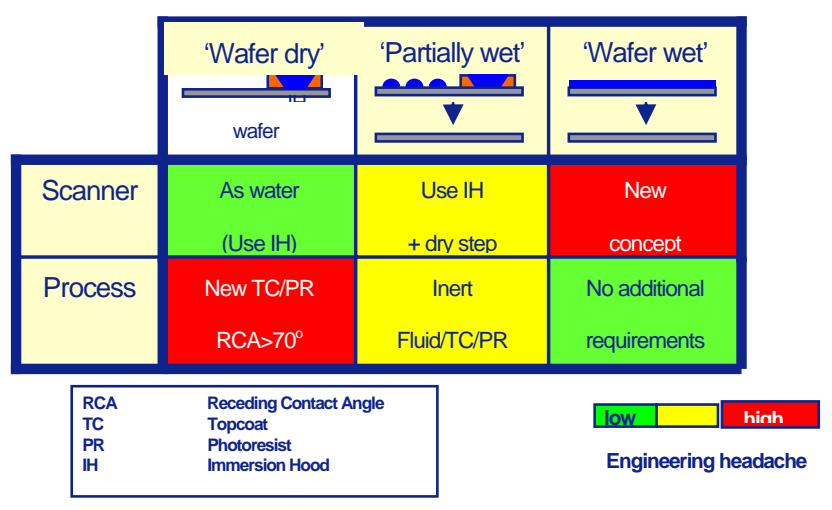

Fig. 1. System Concept Options

Dynamic contact angle measurements made on a scanning test bed for High-n immersion fluid testing reveal a significantly different situation over that of water. High-n immersion fluid has a significantly lower contact angle on resist and on topcoat surfaces than does water. This forces the review of fluid containment options as summarized in Fig. 1. The currently used concept for water based systems is the "wafer dry", but this may not be viable for high-n immersion systems unless new top coat materials can be developed that can significantly increase the contact angle with the high-n fluids. The possibility of using a "wafer wet" concept is being explored, but this too is very challenging to implement. This leads to focus on the "partially wet" concept. For this option the impact on defect counts and the effect of fluid droplets is the major consideration.

\section{Fluid Handling}

The immersion fluid development efforts by fluid vendors such as DuPont ${ }^{1,2}$ and JSR ${ }^{3,4}$ have been very successful. Current fluid samples with a refractive index of about $1.65^{5}$ are readily available. Each supplier has several types of fluid for evaluation. They have made considerable progress towards improving the transparency of their fluids. Fig. 2 shows the absorption values measured on fluids from at two suppliers.

Currently, fluids with a transparency higher than that of water are being delivered for testing. It is noted that the "intrinsic" transparency of the fluid has not yet been reached; improvements to the fluid transparency may

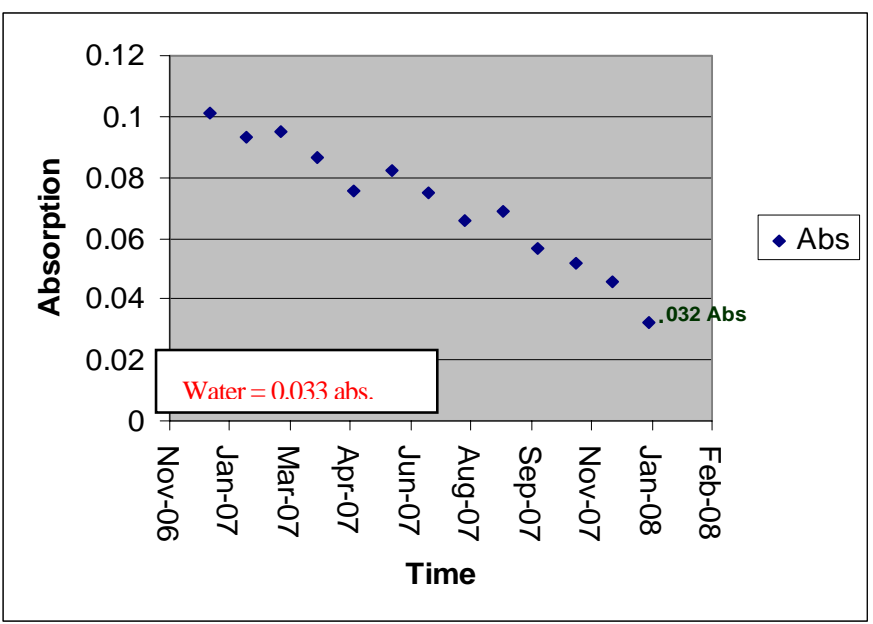

Fig. 2. Improvements in immersion fluid transparency at 193nm wavelength

therefore be continued. The delivered fluid samples are used in UV irradiation testing aimed at characterizing the fluid for application on an exposure tool. Fluid is circulated through a rudimentary fluid-handling system that pumps, filters, de-oxygenates, and reconditions the fluid. The recirculation system typically removes Oxygen that is dissolved in the fluid by displacing it with Nitrogen (Oxygen in the fluid significantly reduces fluid transparency). The system also degasses the fluid.

In the recirculation loop, there is a laser irradiated flow cell with quartz windows (see Fig. 3) and a multipathlength metrology cell for fluid absorption measurement.

\section{Fluid Irradiation Tests}

Fig. 3 shows typical irradiation results where the fluid background absorption is degraded from $0.054 / \mathrm{cm}$ to $0.057 / \mathrm{cm}$ over a three hour irradiation test at $0.8 \mathrm{~mJ} / \mathrm{pulse}$, $100 \mathrm{~Hz}, 1$ liter volume of fluid. The fluid is degraded by the 193nm photon irradiation. The Hydrogen/Carbon bonding is disrupted by photon absorption. Measurements indicate that the process is sensitive to 


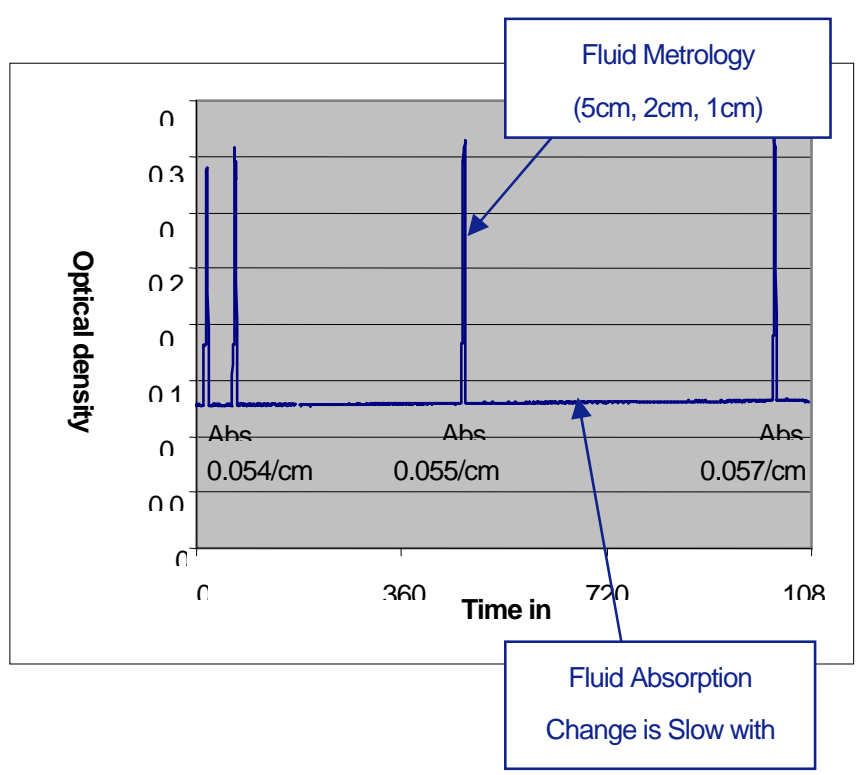

Fluid LifetimeTest: Laser @ 1.6 mJ/cm2; $0.8 \mathrm{~mJ} /$ pulse, $100 \mathrm{~Hz}$, Fluid A.

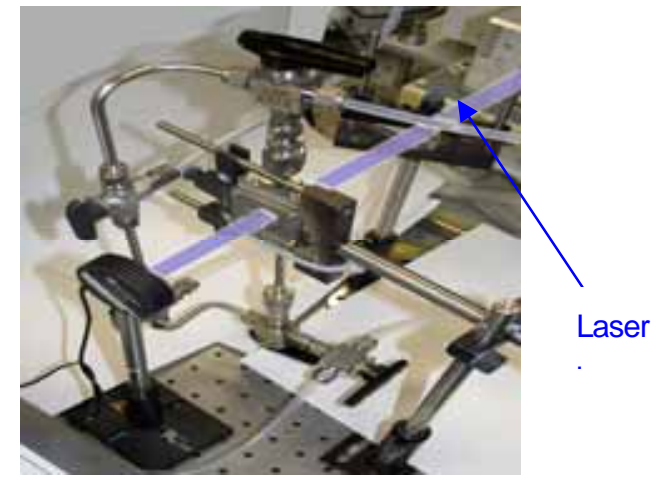

Fig. 3. Irradiation testing of fluids at 193nm wavelength

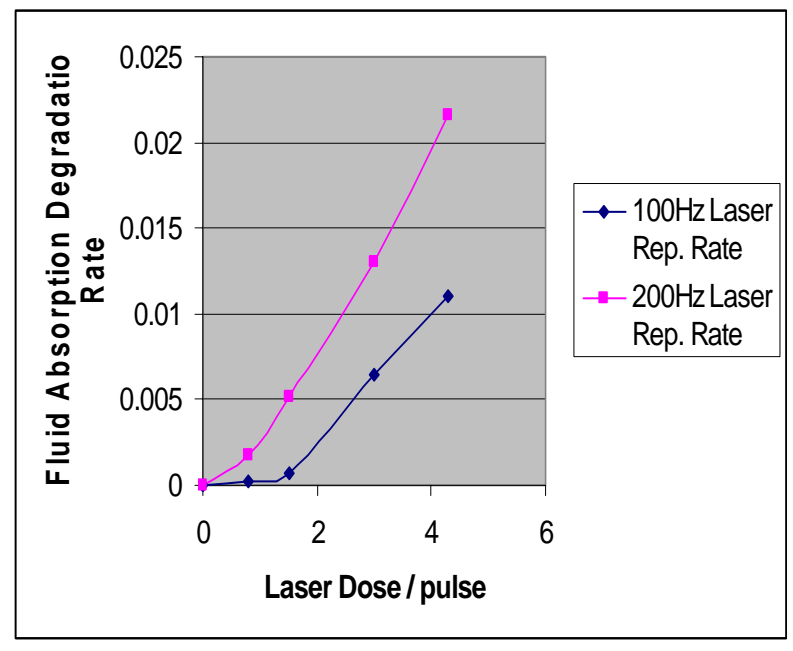

Fig. 4. Fluid degradation rate against laser dose/pulse

laser dose and to the absorption value of the fluid. Fig. 4 indicates that reducing the laser dose per pulse to less than $0.8 \mathrm{~mJ} /$ pulse significantly reduces the fluid degradation rate and, hence, lengthens fluid lifetime.
The fluid degradation is the instantaneous increase in fluid absorbance $/ \mathrm{cm}$ measured for a $200 \mathrm{~mL} / \mathrm{min}$ fluid stream when laser exposure is turned on. This absorbance increase is measured for a range of laser doses/pulse.

Testing also revealed that the rate of degradation of the high transparency fluid is less than that of an absorbing fluid. The achieved improvements in fluid transparency therefore lengthen fluid life. Fluid life is also being improved by the continued development of fluid recycle packages by fluid vendors. Test results from vendor supplied fluid reconditioning packages provide estimates of fluid life in a production system. Estimates indicate that the current 200 liter fluid batch life is expected to be up to ten days on a TWINSCAN ${ }^{\mathrm{TM}}$ operated at typical exposures and wafer throughputs. Further developments in recycle package technology are expected to improve this further.

\section{Final Lens Element Contamination}

An issue with the use of hydrocarbon based immersion fluid is the contamination of the final lens element's surface by carbonaceous deposits from UV degraded fluid ${ }^{6}$. Damaged hydrocarbon- molecules that stick to the lens surface are further degraded and reduced to a carbon rich film. We have developed models of the degradation process to better understand the process and to determine viable mitigation strategies. Figure 5 shows a typical Mass Balance fluid transport analysis.

From this analysis we calculate the velocity profile for the fluid flowing adjacent to the lens surface. It shows that at the lens surface the fluid motion is dominated by the diffusion of the fluid molecules, rather than lateral flow. Window contamination takes place when the rate of photon exposure exceeds the rate at which damaged molecules diffuse away from the lens surface and are swept away in the fluid flow.

Fig. 6 shows a photograph of typical lens contamination deposits from the flow cell experiments. The EDX analysis confirms that it is primarily carbon.

In addition to attempting to mitigate the window contamination, window cleaning processes were studied and evaluated. Test results indicate that with an allowed $2 \%$ lens transmission change specification, the final lens element will have to be cleaned every twenty four hours. 


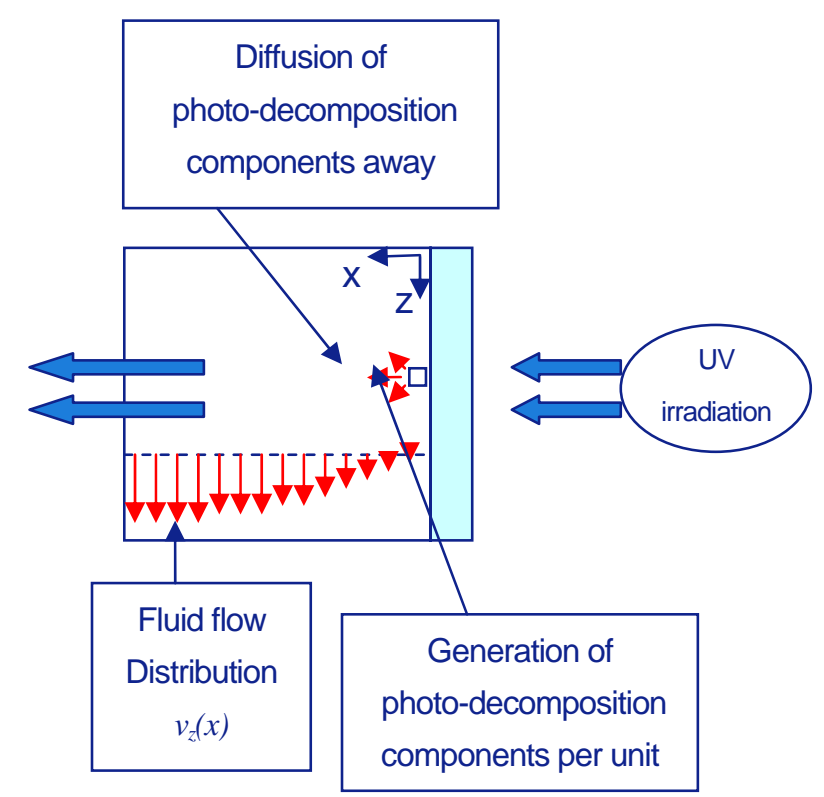

From Mass Balance analysis:

$v_{z}(\max )\left[1-\frac{(\delta-x)^{2}}{\delta^{2}}\right] \frac{\partial c_{a}}{\partial z}+k_{r} \cdot c_{a}=D_{a b} \frac{\partial^{2} c_{a}}{\partial x^{2}}$

Solved for $c_{a}$ at $x=0$

(the window surface) for the following boundary conditions:

$$
\begin{aligned}
& \text { At: } \quad z=0 \quad c_{a}=0 \\
& x=0 \quad v_{z}=0 \\
& x=\delta \quad v_{z}=v_{z}(\max ) \quad \text { and } \quad \frac{\partial c_{a}}{\partial x}=0
\end{aligned}
$$

(fluid flow dominated)

$D_{a b}$ is the Diffusion coefficient of the photo-product $a$ in

fluid $b$.

$k_{r}$ is the Reaction coefficient for fluid breakdown under

UV irradiation. Function (laser).

Fig. 5. Simulation calculations for exposure window contamination

Cleaning process evaluations indicate that the use of an automated UV-O cleaning process can clean the lens in a few minutes. Fig. 7 demonstrates a test cleaning cycle during which the flow cell is irradiated with a high dose to produce a deposit on the cell windows, the fluid is pumped out of the cell, the cell is dried, air with UV illumination is introduced, and the window contamination is removed. In the test, the process
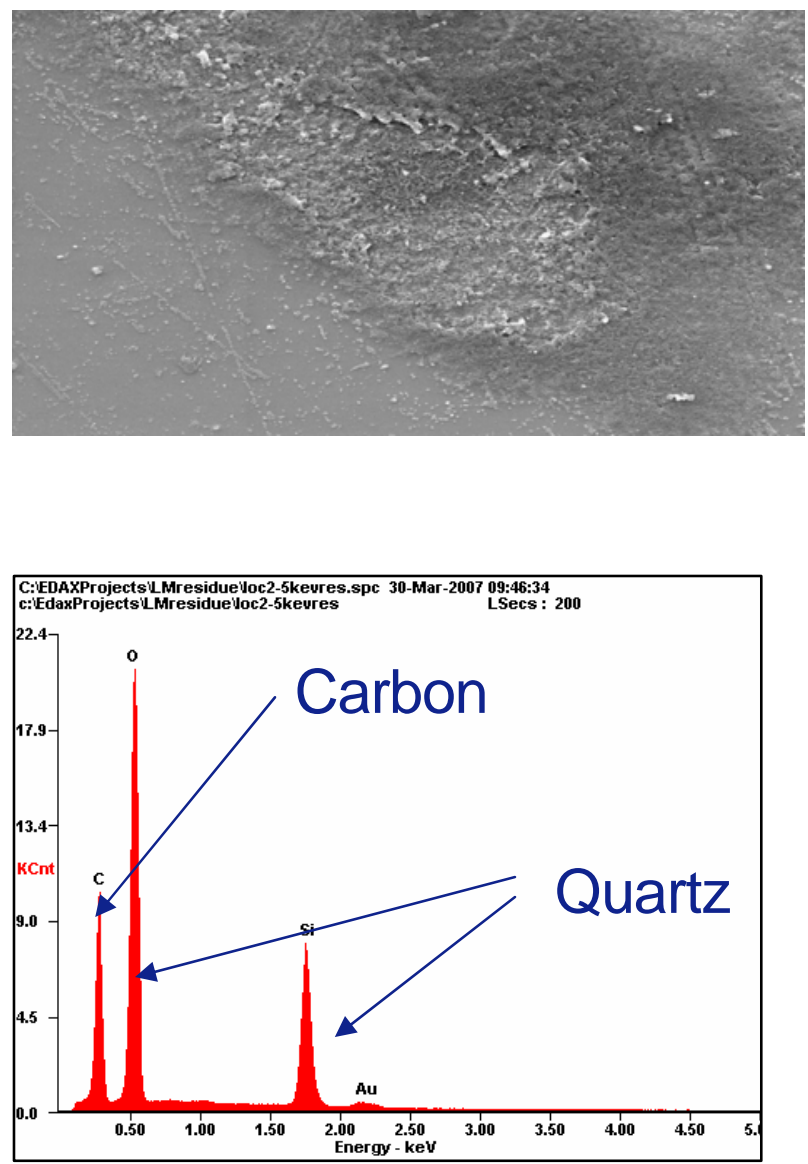

EDX Window Data (Au; sample coating) (Enerqy Dispersive Spectroscopy)

Fig. 6. Contamination on Optics Surface

sequence was cycled a number of times to check its viability. A target for the study of the contamination of the final lens element is to increase the projected times between optics cleaning to more than a month. An increase in time between cleans is expected to be achieved by improving fluid recycling efficiency, fluid flow dynamics, and surface coatings.

Window

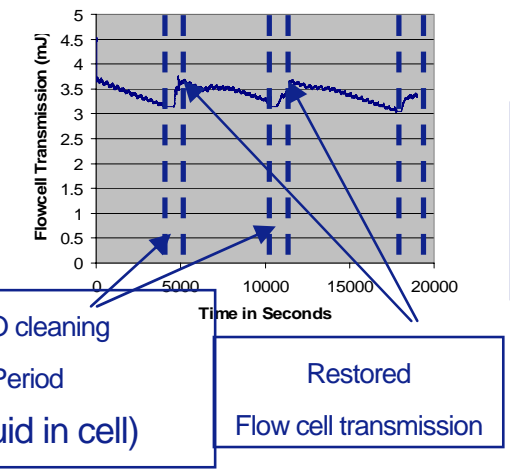

Contamination

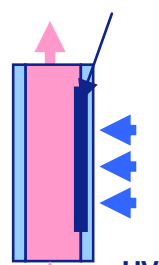

Fluid or

Fig. 7. UV-O cleaning cycle test 


\section{Defects on wafers}

A significant concern with high index immersion lithography is defects on the printed wafers. The feasibility study is looking at defect generation for High$\mathrm{n}$ immersion fluid and comparing it with dry exposed wafers and water based immersion wafers.

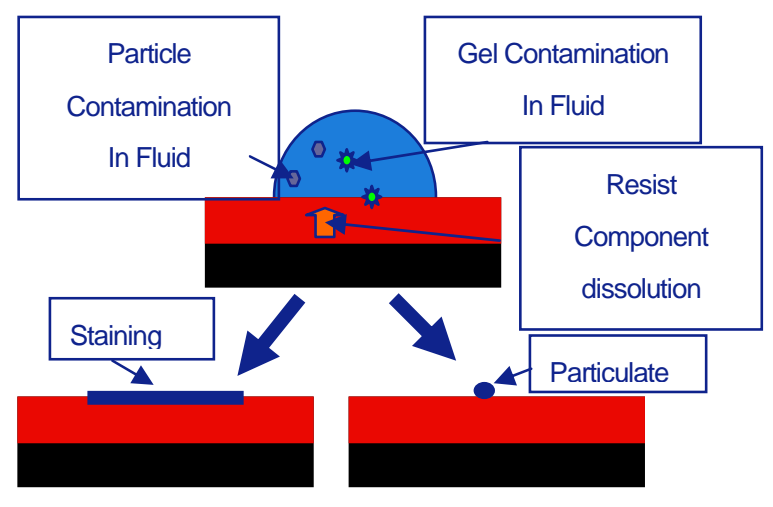

Fig. 8. Defect sources from fluid application to patterned wafers

Typical defect sources from High-n immersion fluid on patterned resist coated wafers are shown in Figure 8.

The defect sources studied include: Fluid drying effects; Particulates in the fluid; Dissolved impurities in the fluid ${ }^{7}$; and interaction products from resist and topcoat $^{8,9,10}$.

\subsection{Fluid Drying Tests}

Two short-term goals have been pursued; 1) To apply a drop of fluid to a plain silicon wafer and have it dry deposit free; 2) To track dispense fluid onto a plain silicon wafer and spin-dry defect-free. Some defects are fluid handling related and some are fluid cleanliness related. Typically, the fluid supplied by vendors must be filtered to remove particulates.

The particulates can come from the fluid recycle columns that are used to purify the fluid. Figure 9 indicates test results from experiments with CUNO NanoSHIELD Teflon filter cartridge.

The count for particles $(>0.05 \mathrm{um})$ was reduced from $47,000 / \mathrm{ml}$ to just $37 / \mathrm{ml}$ by using a single-stage filter; this produced a matching reduction of defects on a plain silicon test wafer. With the use of any particle filter it is important to make sure that the materials used in the construction of the filter are compatible with the High-n immersion fluid and do not cause chemical contamination.

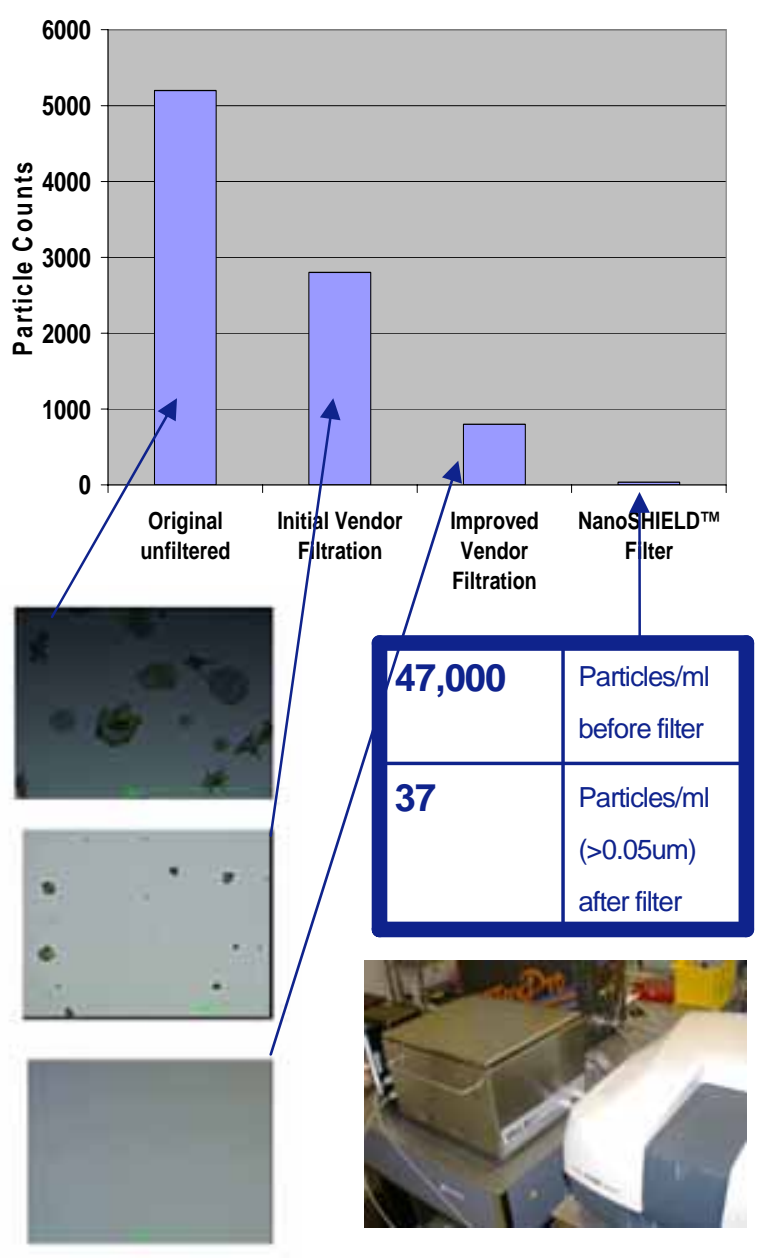

Fig. 9. Fluid filtering and Drop Drying test of High-n immersion fluid

\subsection{Materials of Construction Tests}

Part of the challenge to produce defect-free dropdrying test results is to ensure that the immersion fluid is not contaminated by materials of construction used for the test apparatus and exposure tool. All construction materials must be screened and tested. We have developed a test in which material samples are soaked in clean immersion fluid for one day and five days. The immersion fluid absorbance value is monitored. Any change in fluid absorbance will fail the material under test. Tests like this have been used to qualify degasser membrane samples with companies such as Entegris. Besides materials of construction there can be other sources of dissolved material. Resist and top coat on the wafers are potentially prime sources.

\subsection{Resist Fluid Interactions}

CD Uniformity measurements and Contrast Curve tests have been run to study fluid/resist interactions. Figure 10 shows Contrast Curve test results for two resists, AR1682J and AM2073J. In this test, resist 
thickness after development is plotted for the different doses used in the exposure matrix. The resist contrast can be calculated from the slope of the curves. Fluid is applied to the exposed area and the effects on the shape of the contrast curve are observed. Resist behaviors differ slightly from one to the other and with the different fluid versions. The results depend on the particular resist type and indicate whether there is any interaction between the resist and the fluid. The main interaction with the fluid, if any, is observed with the exposed resist.
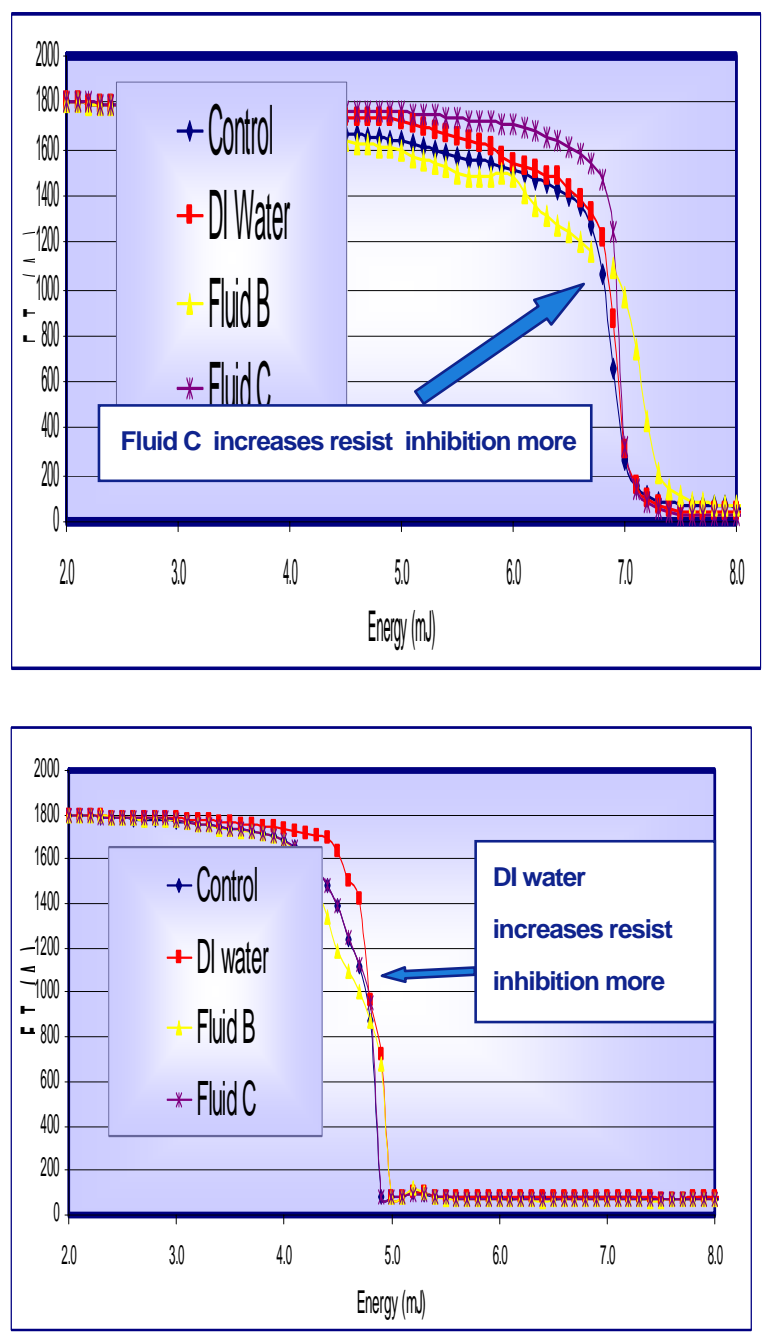

Fig. 10. Resist Contrast Curves for testing fluid resist interactions

\section{Optics}

The optics in a High-n immersion system is a key component. Design reviews with Zeiss, indicate that the current in-line catadioptric lens concept used in TWINSCAN $^{\mathrm{TM}}$ XT1700i and XT1900i systems, can be extended to higher NA. With high index glass as last lens element the design can be extended to numerical apertures of 1.55. Significant efforts are therefore being made to develop suitable optical materials for the final lens element. The growing and testing of high index glass materials has been under way for some time, and currently, the most progress is being made in the development of LuAG material with a refractive index of 2.1. The key parameter that requires improvement is the absorption coefficient of the material at 193nm. This optical absorption value is dominated by the presence of impurities such as $\mathrm{Fe}$ and $\mathrm{Cr}$ in the raw materials. A broad range of refining and purification techniques are being harnessed to reduce impurities to the parts per billion and to bring the absorption coefficient down to the optical material specification level. Figure 11 indicates the progress being made at Schott for their work with LuAG. The latest results presented at SPIE 2008, report the achievement of $0.03 / \mathrm{cm}-0.05 / \mathrm{cm}$. absorption values. It is expected that new, tighter, feasibility targets $(<0.01 / \mathrm{cm}$.) for absorption will be surpassed later this year (2008).

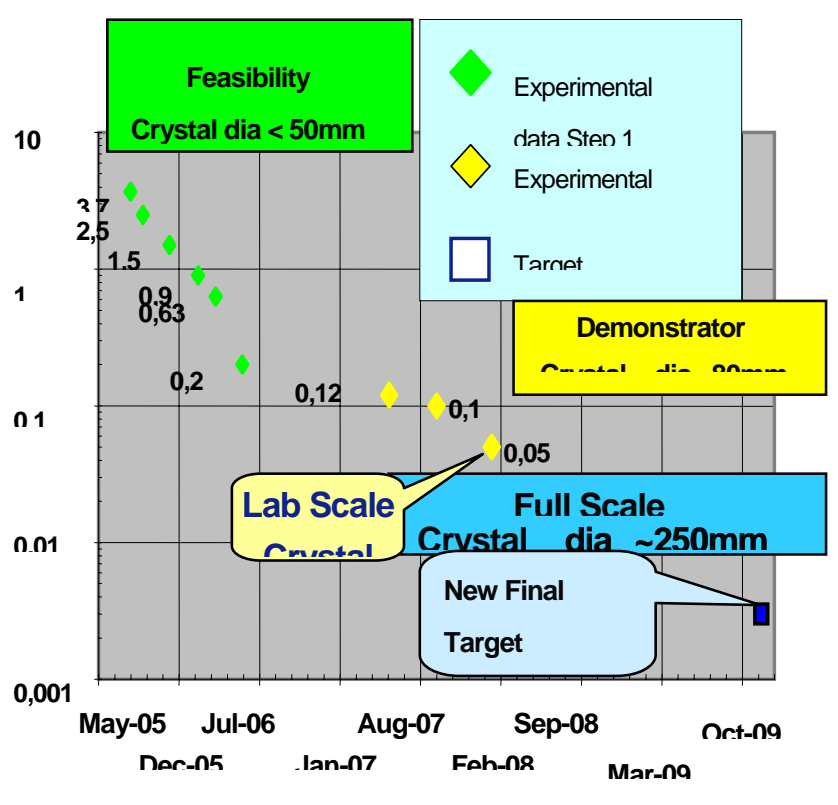

Fig. 11. Absorbance measured in growth tests for LuAG optical material

The issue of optical material availability for the final lens element fabrication is the only technology road block encountered in the feasibility study. Progress toward obtaining optical quality material is being keenly monitored. 


\section{Summary and Conclusions}

The system opportunities that have been identified for the extension of optical immersion lithography by using High-n immersion fluids are indicated in Table 1.

\begin{tabular}{|c|c|c|}
\hline & $N A=1.55$ & NA 1.70 \\
\hline shrink & $15 \%$ & $25 \%$ \\
\hline Technology & $N A=1.55$ & NA 1.70 \\
\hline Optics & ok & ok \\
\hline Lens & \multicolumn{2}{|c|}{ 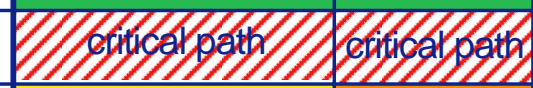 } \\
\hline Fluid & cleanliness & unlikely \\
\hline Resist & no showstopper & high index \\
\hline Exposure & feasible & feasible \\
\hline
\end{tabular}

- Progress on "glass" is slow.

- Gen-3 Fluids need more attention.

Table 1. Technology options in terms of tool opportunities

Progress with High-n immersion lithography feasibility has been steady, and there has been progress in all areas of the technology. Scanning test results indicate that an all wet wafer system solution may be required. Final lens element contamination is a major concern and improvement is required, either in the reduction of the deposition rate or cleaning procedure.

Wafer contamination and final lens element contamination are major issues, but there remains one roadblock issue, the availability of production quality optical material for the final lens element. The projected availability of this material is now the end of 2009, and consequently a first exposure system will not be available before 2011.

It is noted that the extension of the technology to $>1.7$ NA will require a very significant development efforts of Generation-3 fluids and of High-n resists. If this is to happen, there will need to be a significant escalation in the development effort in these areas.

\section{Acknowledgements}

The authors acknowledge support in this work from the following:

ASML-Wilton: Mark Riggs, Bob Pattacini, Matt Lipson, Chris Dadarria, Charlie Griffing,

ASML-Veldhoven: Rogier Cortie,

Ralph Meijers, Fabrizio Evangelista,

ASML-Sunnyvale: Paul Yick, Michael Robles, Arnout Smit,

ASML-Tempe: Donis Flagello, Bill Arnold,

Zeiss-SMT: Ruediger Duesing, Wilfried Clauss,

DuPont: Roger French, John Schmeig, Hoang V. Tran,

JSR: Katsuhiko Hieda, Mark Slezak, Zhi Lui,

Entegris: Bipin Parekh.

\section{References}

1. Roger H.French, Weiming Qiu, et.al.,'Second generation fluids for 193nm immersion lithography," Proc. SPIE 6154, (2006).

2. Roger H.French, V.Liberman, et.al., "High-index immersion lithography with second-generation immersion fluids to enable numerical apertures of 1.55 for cost effective 32-nm half pitches,” Proc. SPIE 6520 (2007).

3. Taiichi Furukawa, Katsuhiko Hieda, et.al.,"Highrefractive index material design for $\mathrm{ArF}$ immersion lithography,” Proc. SPIE 6519 (2007).

4. Yong Wang, Takashi Miyamatsu, et.al.,"Highrefractive-index fluids for the next-generation $\mathrm{ArF}$ immersion lithography," Proc. SPIE 6153 (2006).

5. Roger H.French, Min K.Yang, et.al.,’'Immersion fluid refractive indices using prism minimum deviation techniques,” Proc. SPIE 5377 (2004).

6. V.Liberman, M.Rothschild, et.al.,’Laser durability studies of high index immersion fluids: fluid degradation and optics contamination effects," Proc. SPIE 6520 (2007).

7. V.Liberman, M.Rothschild, et.al., "Impact of photoacid generator leaching on optics photocontamination in 193nm immersion lithography," MEMS MOEMS 6, (2007).

8. Hiroki Nakagawa, Atsushi Nakamura, et.al., "Improvement of watermark defect in immersion lithography: mechanisms of watermark defect formation and its reduction by using alkaline soluble immersion topcoat," Proc. SPIE 6153 (2006).

9. Katsushi Nakano, Hiroshi Kato, et.al., "Immersion defectivity study with volume production immersion lithography tool,” Proc. SPIE 6520 (2007). 
10. Tadatoshi Tomita, Takeshi Shimoaoki, et.al., "An investigation on defect-generation conditions in immersion lithography," Proc. SPIE 6153 (2006).

11. Steven R.Brueck, Abani M.Biswas, "Extension of 193-nm immersion optical lithography to the 22-nm half-pitch node," Proc. SPIE 5377 (2004).
12. A.Vanleenhove, D.Van Steenwinckel, "A litho-only approach to double patterning," Proc. SPIE 6520 (2007). 